\title{
Tomografía computarizada de haz cónico como examen concluyente de hiperplasia de procesos coronoides bilateral. Reporte de tres casos
}

Cone beam computed tomography as a conclusive examination of bilateral coronoid process hyperplasia. Report of three cases

Carlos Valenzuela ${ }^{1 a}$, Joseline Fritz ${ }^{1, a}$, Alejandro Schilling ${ }^{1, a, b}$, César Celis ${ }^{1, a, b}$, Alejandro Hidalgo ${ }^{1, a, b, c}$. RESUMEN

La hiperplasia del proceso coronoides (HPC) es un crecimiento exagerado del proceso coronoides. La HPC es poco frecuente y genera limitación asintomática de la apertura bucal debido a la impactación del proceso coronoides con el segmento posterior del hueso cigomático. La HPC es un posible diagnóstico en pacientes con limitación progresiva tanto de la apertura bucal como del movimiento protrusivo mandibular. En el presente artículo, se reportan tres casos de pacientes con limitación de apertura bucal. Mediante examen de tomografía computarizada de haz cónico (TCHC), se observó un aumento de tamaño uniforme de los procesos coronoides, confirmando el diagnóstico de HPC bilateral. La TCHC con campo de visión grande determinó el diagnóstico final, pues mostró la impactación del proceso coronoides con el segmento posterior del hueso cigomático en apertura bucal. Además, la TCHC permitió distinguir entre una HPC y una neoplasia del proceso coronoides y/o estructuras anatómicas vecinas.

PALABRAS CLAVES: Tomografía computarizada de haz cónico, hiperplasia, reporte de casos.

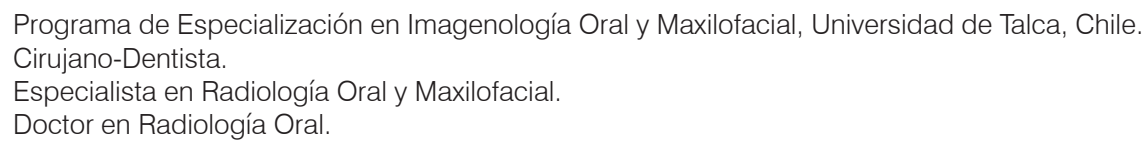




\section{SUMMARY}

Coronoid process hyperplasia $(\mathrm{CPH})$ is an excessive coronoid process growing. $\mathrm{CPH}$ is infrequent and produces an asymptomatic mouth opening limitation due to the impaction of the coronoid process in the posterior portion of the zygomatic bone. $\mathrm{CPH}$ is a possible diagnosis in patients with both progressive mouth opening and protrusive movement limitations. In the present article, cases of three patients with mouth opening limitation are reported. In cone-beam computed tomography (CBCT) examination, a uniform growing of coronoid processes was observed, which confirmed the diagnosis of bilateral CPH. CBCT with large field of view allowed the determination of the final diagnosis, because the impaction of the coronoid process in the posterior portion of the zygomatic bone during mouth opening could be observed. Furthermore, CBCT allowed to distinguish between $\mathrm{CPH}$ and neoplasia of coronoid process and/or neighboring anatomical structures.

KEY WORDS: Cone-beam computed tomography, hyperplasia, case reports.

\section{INTRODUCCIÓN}

La altura del proceso coronoides (PC) mandibular normalmente es de $12-13 \mathrm{~mm}$, pero en la hiperplasia del proceso coronoides (HPC), puede medir sobre 20 mm (1). La HPC se diagnostica en el 5\% de los casos que presentan limitación en la dinámica mandibular (2) y corresponde a un crecimiento exagerado en altura y volumen del PC (3). Este crecimiento es asintomático e histológicamente corresponde a hueso normal (4).

La HPC genera una limitación progresiva tanto de la apertura bucal como de protrusión mandibular (5). Esta limitación en la dinámica mandibular, se debe a la impactación del PC hiperplásico con el segmento posterior del hueso cigomático (2). La HPC puede ser unilateral o bilateral, siendo los casos HPC bilateral cuatro veces más frecuentes que los unilaterales (6). En casos de HPC, los exámenes imagenológicos muestran generalmente la articulación temporomandibular (ATM) normal. Sin embargo, el PC es de mayor tamaño, pudiendo sobrepasar en altura al arco cigomático (5). La evaluación inicial de una HPC puede ser una radiografía panorámica, aunque no siempre es un examen concluyente (1).

Si bien la HPC se ha descrito previamente (3) y existen reportes de serie de casos $(6,7)$, no se encontró series de casos de HPC bilateral con hipótesis diagnóstica de anquilosis de ATM, evaluados con tomografía computarizada de haz cónico (TCHC). El objetivo de la presente publicación es reportar tres casos con hipótesis diagnóstica de anquilosis de ATM, descartada mediante TCHC y con diagnóstico final de HPC.

\section{Reporte de casos}

Se reportan tres casos de pacientes de género femenino, de 25,58 y 15 años respectivamente, derivadas a un centro radiológico maxilofacial privado de Talca, Chile. Las tres pacientes presentaban limitación de apertura bucal, con hipótesis diagnóstica de anquilosis de ATM.

A las pacientes se les solicitó un examen de TCHC para evaluar el estado de las ATM. Se realizó adquisición volumétrica con equipo SkyView (MyRay, Imola, Italia), con 9 pulgadas (") de campo de visión (FOV, del inglés field of view) y $0,33 \mathrm{~mm}$ de tamaño de vóxel. Se reconstruyó multiplanarmente mediante software SkyView (MyRay, Imola, Italia) obteniéndose imágenes en el plano sagital corregido con espesor de $15 \mathrm{~mm}$ (figura 1), axial (figura 2) y modo reconstrucción $3 \mathrm{D}$.

En todos los casos presentados, la reconstrucción 3D a boca cerrada mostró un aumento uniforme de tamaño del PC bilateralmente, sobrepasando el límite superior del arco cigomático, con características óseas normales y morfología conservada. (figura 3(A) y figura (A y B)). En la reconstrucción 3D a boca abierta se observó la impactación del PC en el segmento posterior del hueso cigomático (figura 3 (B)), impactando en la pared posterior de la fosa infratemporal, por medial al arco cigomático. Por tanto, en todos los casos se descartó la hipótesis diagnóstica de anquilosis de ATM y se confirmó el diagnóstico de HPC bilateral.

\section{DISCUSIÓN}



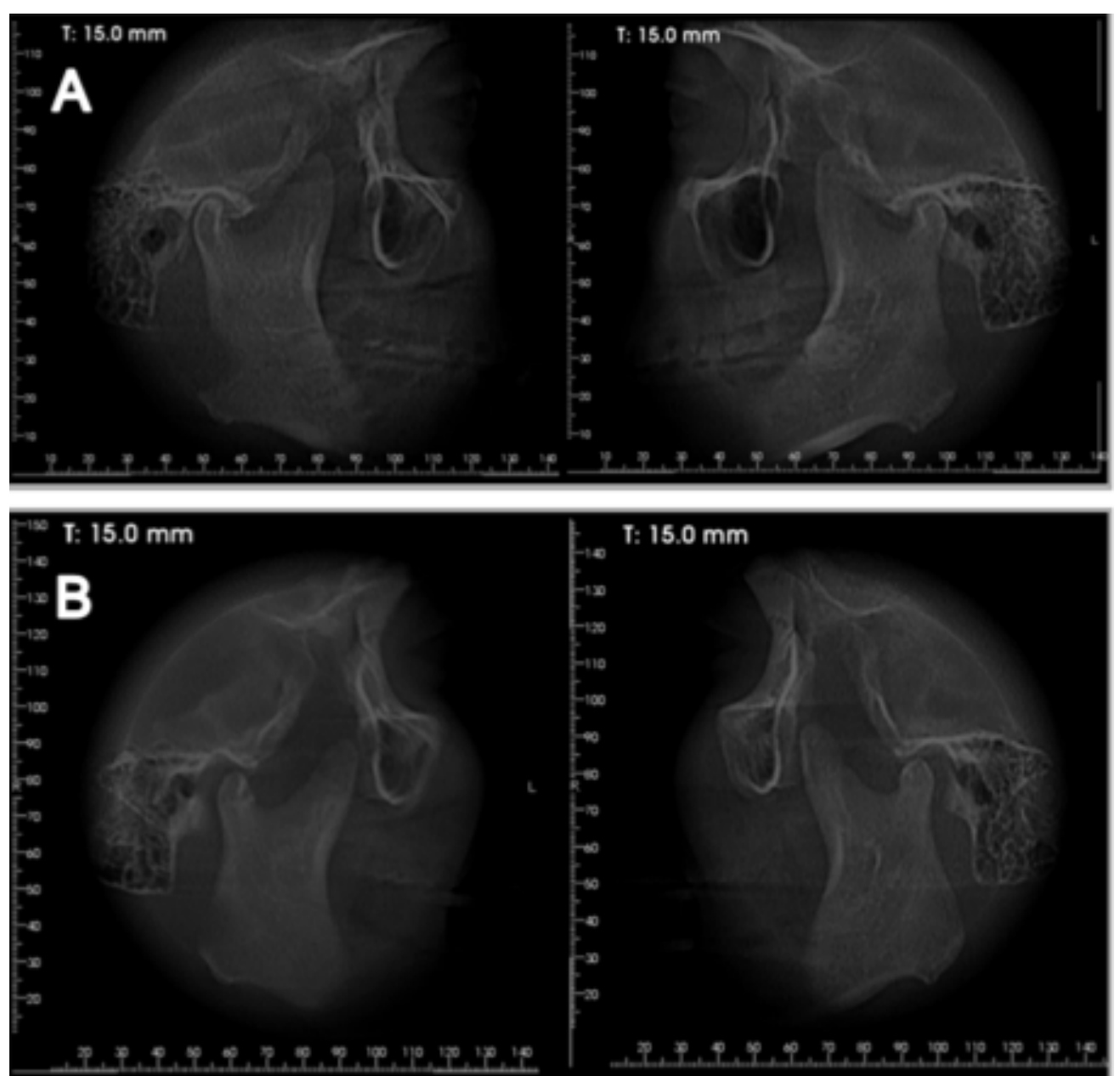

Figura 1. Paciente de 25 años. Plano sagital corregido de tomografía computarizada de haz cónico. Hiperplasia del proceso coronoides bilateral a boca cerrada (A) y a boca abierta (B). (A) se observa mayor altura de los procesos coronoides, sobrepasando arcos cigomáticos bilateralmente en adquisición volumétrica a boca cerrada. (B) se observa impactación del proceso coronoides con el segmento posterior del hueso cigomático, a nivel de pared anterior de la fosa infratemporal. El proceso coronoides triplica la altura del cóndilo mandibular.
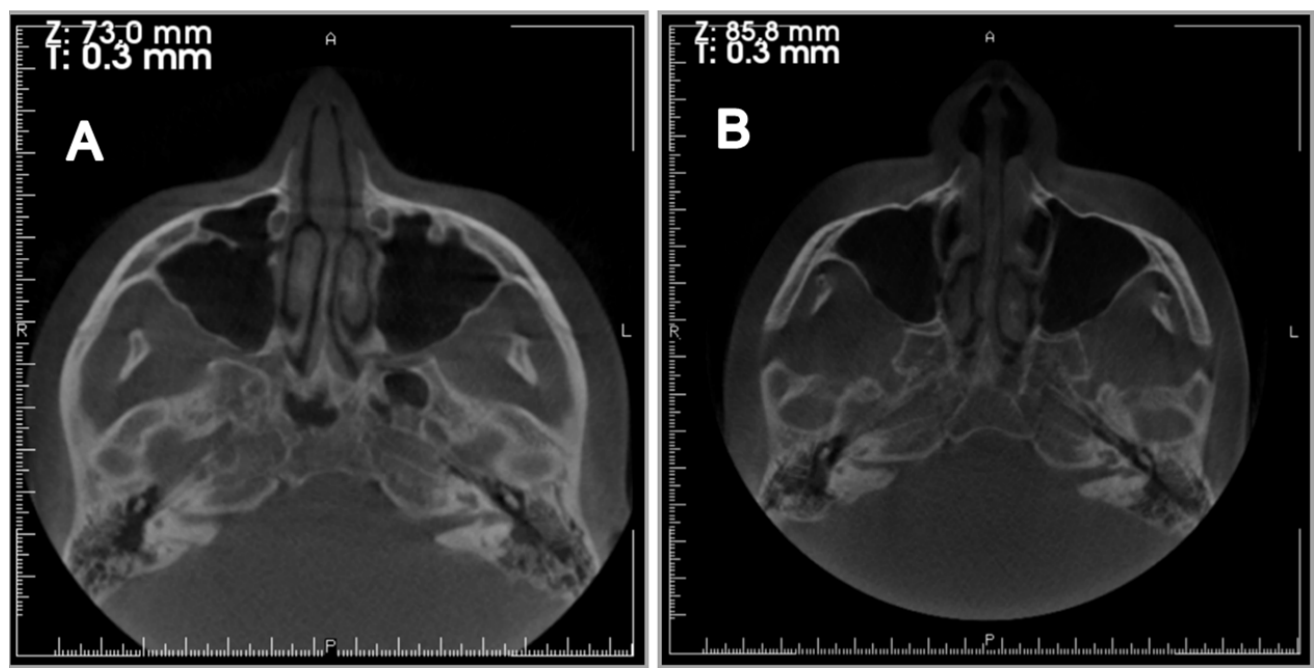

Figura 2. Paciente de 25 años. Plano axial de tomografía computarizada de haz cónico por sobre el nivel condilar. Hiperplasia del proceso coronoides bilateral a boca cerrada (A) y a boca abierta (B). Los procesos coronoides no se observan normalmente a este nivel. 

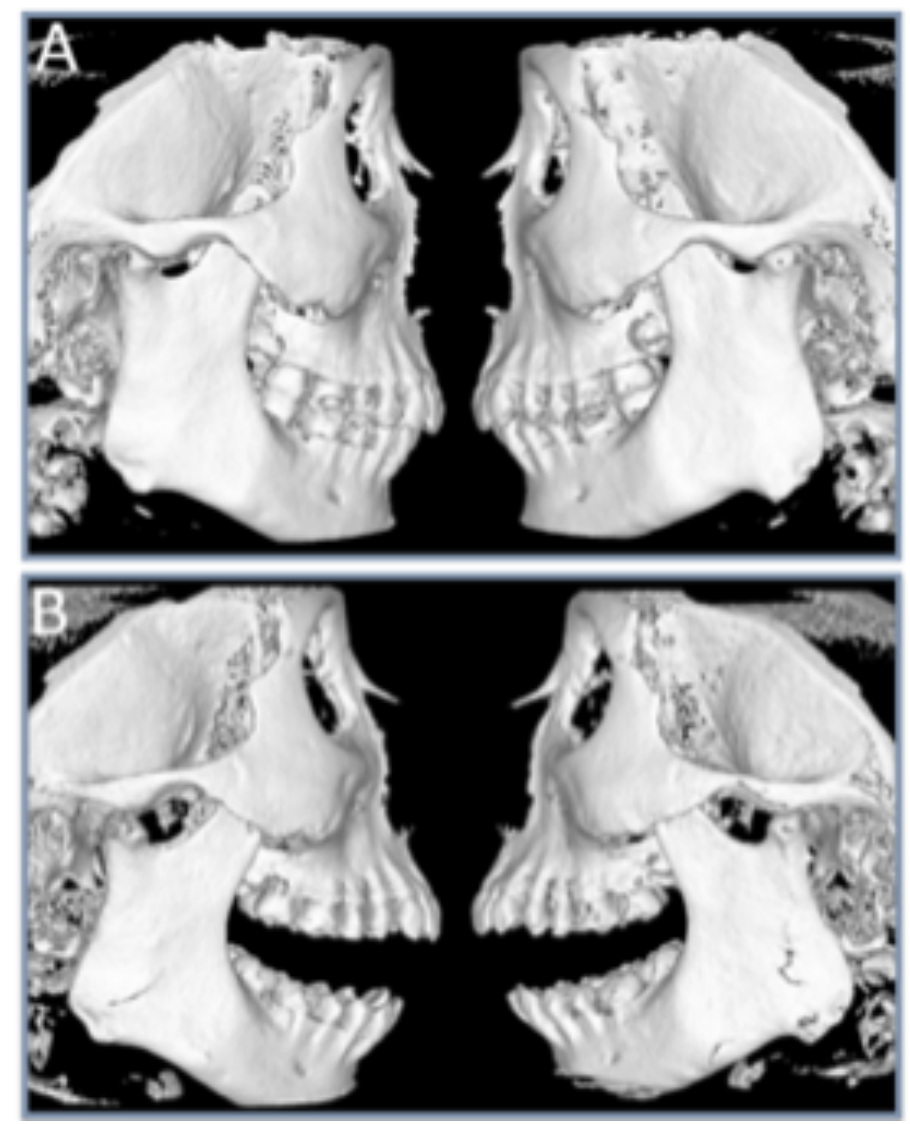

Figura 3. Paciente de 25 años. Reconstrucción tridimensional de hiperplasia del proceso coronoides bilateral. A) Posición proceso coronoides boca cerrada y B) Posición proceso coronoides boca abierta. En reconstrucción a boca cerrada el proceso coronoides sobrepasa el límite superior del arco cigomático bilateralmente.
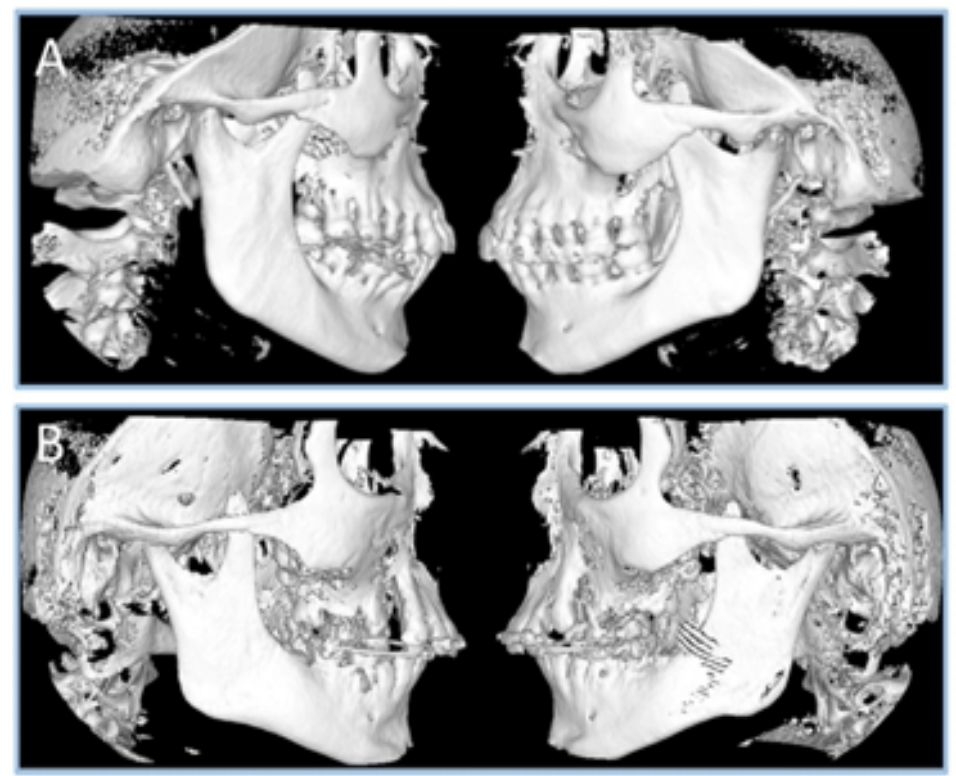

Figura 4. A) Paciente de 58 años y B) de 15 años. Reconstrucción tridimensional de hiperplasia del proceso coronoides bilateral a boca cerrada. En ambos casos, el proceso coronoides supera bilateralmente al límite superior del arco cigomático. 


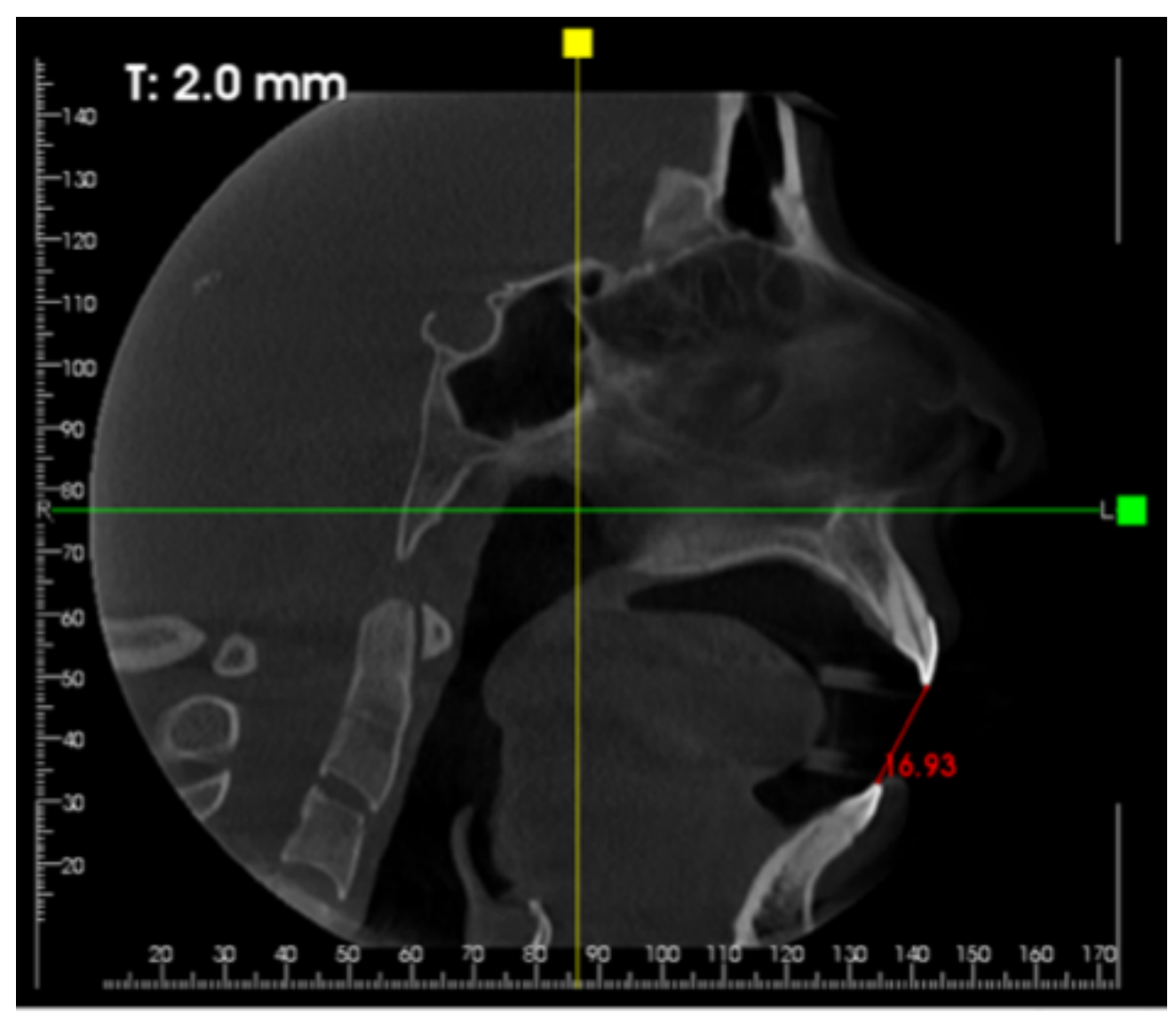

Figura 5. Paciente de 25 años. Plano sagital de tomografía computarizada de haz cónico. Medición de la apertura bucal $(16,93 \mathrm{~mm})$.

El presente reporte muestra la importancia de la TCHC en el diagnóstico de HPC. Los casos reportados tenían hipótesis diagnóstica de anquilosis de ATM, producto de la marcada limitación de apertura al examen clínico. La HPC puede encontrarse hasta en el 5\% de los pacientes con síntomas de la ATM y con limitación de la apertura bucal (8). En TCHC con FOV grande se observó los componentes óseos articulares y extra-articulares, estableciendo la relación del PC con estructuras óseas adyacentes en apertura bucal, lo que permitió el diagnóstico final de HPC.

Los tres pacientes reportados eran mujeres, lo que no concuerda con la literatura, que señala que la HPC afecta principalmente al género masculino $(5: 1)$ (2). La edad media al momento del diagnóstico de HPC es 25,5 años, con un rango de mayor frecuencia de 20 a 30 años (3). En el presente reporte, sólo un caso (25 años) coincide con dicho rango de edad. Los otros casos se diagnosticaron previo (15 años) y posterior (58 años) a dicho rango. El diagnóstico a los
58 años de uno de los casos, posiblemente indica un diagnóstico clínico errado de larga data.

La apertura bucal máxima (ABM) en normalidad es de 40-60 mm (7), considerando como disminución significativa si ésta es inferior a $35 \mathrm{~mm}$ (9). Se ha reportado una media de ABM en HPC sin tratamiento de 18,5 mm (3). Si bien en el presente reporte no tenía mediciones clínicas de ABM, ésta fue de 16,9 mm en las imágenes de TCHC de la paciente de 25 años (figura 5).

El diagnóstico clínico de HPC suele ser difícil, por la tendencia a atribuir sus signos y síntomas a trastornos de ATM $(2,7)$. La evolución de la limitación de apertura bucal en HPC es progresiva, indolora para el paciente (3) y no altera la oclusión (1). Sin embargo puede ocasionar retardo en el crecimiento mandibular (1) y atrofia muscular (5). Además, esta limitación de apertura puede dificultar la respiración, alimentación y cepillado de los dientes, lo que implica 
Tabla 1. Alternativas diagnósticas para procesos asintomáticos que producen limitación de apertura bucal y sensación final dura.

\begin{tabular}{cc}
\hline ALTERACIONES DE ATM & CONDICIONES EXTRA-ARTICULARES \\
\hline \multirow{2}{*}{ Anquilosis de ATM } & Hiperplasia de proceso coronoides \\
& Neoplasia de proceso coronoides \\
Neoplasia de hueso cigomático & Enfermedad de Jacob \\
Neoplasia de ATM & Fractura de hueso cigomático mal consolidada \\
& Deformidades óseas próximas a rama mandibular \\
\hline
\end{tabular}

un desafío para el odontólogo en el tratamiento de las potenciales patologías bucodentales al enfrentarse a un área operatoria disminuida (1).

El análisis de los PC con imágenes bidimensionales (2D) también es complejo. La imagen 2D debe considerarse como un paso inicial (4), sin embargo, para el diagnóstico definitivo de HPC se requiere una evaluación 3D.

En el presente reporte, se proponen diagnósticos diferenciales clínicos de HPC en procesos asintomáticos que producen limitación de apertura bucal $(8)$ y sensación final dura $(4,10)$ tanto intra como extra- articulares (10) (Tabla 1). El presente reporte permite enfatizar en la importancia de considerar la evaluación de los PC en la sistemática del diagnóstico imagenológico.

En la radiografía panorámica, la HPC puede verse como una mayor altura del PC en comparación con el cóndilo mandibular. Además, los procesos coronoides sobrepasan el arco cigomático (11). Sin embargo, en estadios iniciales de crecimiento de los PC, la radiografía panorámica puede no aportar suficiente información (5).

En TCHC, el uso de imágenes multiplanares y 3D es fundamental para diagnosticar la HPC, siendo la reconstrucción 3D la más explicativa (12). Las imágenes axiales y sagitales a boca cerrada y abierta, son valiosas en la evaluación de pacientes con sospecha de HPC (8). Además, la reconstrucción 3D permite distinguir entre una HPC y una neoplasia del PC y/o estructuras anatómicas vecinas, pues permite evaluar la morfología ósea con detalle $(4,13)$.
En los casos reportados se evaluó las dos ATM con TCHC usando un FOV grande. El FOV grande ofreció un mayor valor diagnóstico ya que facilitó la evaluación de las dos ATM en conjunto y de condiciones extra-articulares. El FOV grande del equipo de TCHC usado, registra menor dosis de radiación $(96,8 \mu \mathrm{Sv})$ que usar dos $\mathrm{FOV}$ pequeños (4") para evaluar cada ATM por separado $(58,0 \mu \mathrm{Sv}$ x $2=116 \mu \mathrm{Sv}$ ). Así, se cumplió con el principio de protección radiológica de optimización (14).

En conclusión, la HPC es poco frecuente, pero debe considerarse como alternativa diagnóstica en pacientes con limitación principalmente en movimientos de apertura y protrusión. La TCHC a través de sus diferentes planos de imagen y reconstrucción 3D es fundamental para descartar hipótesis diagnósticas de alteraciones de ATM y concluir la HPC.

\section{Correspondencia:}

Alejandro Hidalgo

Av. Lircay S/N, Escuela de Odontología, Universidad de Talca. Talca, Chile

Teléfono: +56 712200476

Correo electrónico: ahidalgo@utalca.cl

\section{REFERENCIAS BIBLIOGRÁFICAS}

1. AcostaC,LorancaG.Hiperplasiadeprocesoscoronoideos mandibulares: un nuevo tratamiento posquirúrgico. Rev Esp Cir Oral Maxilofac. 2015; 37(2):93-98.

2. Ghazizadeh M, Sheikhi M, Mahdi M, Khaleghi A. Bilateral coronoid hyperplasia causing painless limitation of mandibular movement. Radiol Case Rep. 2018; 13(1):112-117. 
3. Estévez O, Leco MI, Martínez N, Barona C, Martínez JM. Hiperplasia de la apófisis coronoides. Revisión bibliográfica. Cient Dent. 2012; 9(1):63-68.

4. Torenek K, Burak S, Sevki I, Miloglu O. Clinical and radiological findings of a bilateral coronoid hyperplasia case. Eur J Dent. 2015; 9(1):149-152.

5. Moretti E, Cal IO, Galetto M, Londoño L, Blanco R. Hipertrofia del proceso coronoideo. Cir Plást Iberolatinoam. 2014; 40(2):171-177.

6. Surianti H, Yuanchien M. Coronoid process hyperplasia-Report of 3 cases. Taiwan J Oral Maxillofac Surg. 2015; 26(4):310-320.

7. Ilguy M, Kursoglu P, Ilguy D. Three cases of elongated mandibular coronoid process with different presentations. Iran J Radiol. 2014; 11(1):e4031.

8. Som PM, Curtin HD. Imagenología de cabeza y cuello. 5ta edición. Caracas: Editorial AMOLCA; 2015. p. 1604-1605.

9. Jenkins S, Smart K, Mustafa S, Evans R. Bilateral mandibular coronoid hyperplasia causing trismus-a case report. Oral Surg. 2016; 9(4):227-233.

10. Eguia del Valle A, Uribarri A, Martínez R, López J, Ginestal E, Aguirre JM. Hiperplasia coronoidea bilateral. Una rara patología causante de disfunción temporomandibular. Av Odontoestomatol. 2011; 27(5):241-244.

11. Çorumlu U, Kopuz C, Tevfik M, Ece M. Bilateral elongated mandibular coronoid process in an Anatolian skull. Anat Cell Biol. 2016; 49(3):217-220.

12. Domingos V, Hernandez A, Freitas C, Fenyo-Pereira M, Raitz R. Perception of professionals in the assessment of coronoid hyperplasia by computed tomography. Acta Odontol. Latinoam. 2015; 28(1):58-63.

13. Popovik D, Benedetti A, Popovski V, et al. Mandibular movement restoration in a child with bilateral coronoid hyperplasia: a case report. J Med Sci. 2016; 4(2):293295.

14. European Commission. Radiation Protection No 172. Cone beam CT for dental and maxillofacial radiology. Evidence-based guidelines. European Commission; 2012.

Recibido : 16-11-2018

Aceptado : 16-12-2020 